\title{
Magnetic-field dependence of the localization length in Anderson insulators
}

\author{
Igor V. Lerner \\ School of Physics and Space Research, University of Birmingham, Edgbaston, Birmingham B15 2TT, United Kingdom \\ Yoseph Imry \\ Weizmann Institute of Science, Department of Condensed Matter Physics, IL-76100 Rehovot, Israel
}

Submitted to Europhysics Letters, July 30, 1994

\begin{abstract}
Using the conventional scaling approach as well as the renormalization group analysis in $d=2+\epsilon$ dimensions, we calculate the localization length $\xi(B)$ in the presence of a magnetic field $B$. For the quasi $1 \mathrm{D}$ case the results are consistent with a universal increase of $\xi(B)$ by a numerical factor when the magnetic field is in the range $\ell \ll \ell_{H} \lesssim \xi(0), \ell$ is the mean free path, $\ell_{H}$ is the magnetic length $\sqrt{\hbar c / e B}$. However, for $d \geq 2$ where the magnetic field does cause delocalization there is no universal relation between $\xi(B)$ and $\xi(0)$. The effect of spin-orbit interaction is briefly considered as well.
\end{abstract}

Different universality classes, introduced by Dyson [1], became the framework for considering the role of symmetry breaking (due to the presence of a magnetic field, or spin-orbit scattering) in transport properties of disordered electron systems. This role has been well understood in the weak-localization regime, and in the vicinity of the Anderson metal-insulator transition [2 4]. On the other hand, the classification in terms of the universality classes appears to be quite useful also for quasione-dimensional systems in the strongly localized regime. One of the intriguing predictions for this regime [5] is the existence of the universal relation, $\xi_{\beta}=\beta \times \xi_{\beta=1}$, between localization lengths in different universality classes characterized by the parameter $\beta$. There is, however, some controversy: while the transfer-matrix approach based on the maximal entropy ansatz [0],8 predicts that $\beta=1,2$, or 4 for orthogonal, unitary, and symplectic ensembles respectively, in accordance with the Dyson's definition for the random-matrix theory [9], the exact calculation within the nonlinear $\sigma$ model [5] shows that were spin-rotation symmetry broken, $\beta$ would be equal 4 even if time-reversal symmetry were broken as well, although such an ensemble would behave as unitary $(\beta=2)$ in relation to the level statistics [5]. Nevertheless, the existence of a universal relation for $\xi$ has been reliably established for quasi-one-dimensional systems, including quantumchaotic ones [10]. However, the extension of the transfermatrix approach to a higher dimensionality, as in Ref. [8], is not obviously justifiable.

In the present paper we analyze the influence of a magnetic field $B$ and spin-orbit scattering (at $B=0$ ) on the localization length $\xi$ applying the standard oneparameter scaling and renormalization group (RG) approach [4]. The advantage of such a simple approach is possibility to apply the very same scheme to any dimensionality, $d$. We show that, while being in a qualitative agreement with the above-mentioned universality for $\xi$ in quasi-1D systems, the scaling predicts the absence of any universal relation for $d \geq 2$.

The RG equation for the dimensionless conductance $g(L)$ is

$$
d \ln g / d \ln L=\beta(g) .
$$

Here $g(L)$ is defined for a $d$-dimensional system as the conductance in units of $\alpha_{d}\left(e^{2} / \pi \hbar\right)$ with $\alpha_{d} \equiv$ $2 S_{d-1} /(2 \pi)^{d}, S_{d}$ being the area of the $d$-dimensional unit sphere. The $\beta$ function depends on the time-reversal and spin-rotational symmetry of the problem. Let us denote the $\beta$ functions at $B=0$, at large $B$ and in the presence of "strong" spin-orbit scattering (at $B=0$ ) respectively as $\beta_{o}, \beta_{u}$ and $\beta_{s}$. The subscripts $o, u$ and $s$ stand for orthogonal, unitary and symplectic symmetries, respectively.

The question is when is the symmetry effectively broken. The answer depends on which length scale, $L$, we are in. We start at the microscopic scale $L \sim \ell$, with $\ell$ being the mean free path, and go up to the range $L \lesssim \xi$. At $d>2$, that includes both localized and extended phases. In the extended phase $\xi$ has the meaning of the correlation length which is indistinguishable in the critical regime, $L \lesssim \xi$, from the localization length in the insulating phase. At $d \leq 2$, localization sets in with $L$ increasing up to and beyond $\xi$.

In a semiclassical approximation, typical electron trajectories have phases $\left(2 \pi \phi / \phi_{0}\right)$ (with $\phi_{0}=h c / e$ being the flux quantum) which are negligible for small fluxes, when $L \ll \ell_{H} \equiv \sqrt{\hbar c / e B}$ and the problem is effectively that of $B=0$. On the other hand, once $B L^{2} \gtrsim \phi_{0}$, the phases are significant and the time-reversal symmetry is effectively broken. As long as localization has not set in, most of the relevant trajectories cover the whole area of the system, and increasing $B$ beyond $\ell_{H} \sim L$ should not further change the symmetry of the problem. Similar considerations may well be valid for general Feynman paths, beyond the semiclassical approximation?. Thus we are led to the following assumption: The correct $\beta(g)$

\footnotetext{
${ }^{1}$ We do not consider here the range of larger magnetic fields $\ell_{H} \lesssim \ell$, where this treatment breaks down as the classical trajectories bend and orbit shrinking starts. So we leave out
} 
(for $g \gtrsim 1, L \lesssim \xi$ ) can be approximated by $\beta_{o}$ for $L \lesssim \ell_{H}$ and by $\beta_{u}$ for $L \gtrsim \ell_{H}$.

Similarly, we assume that for weak spin-orbit scattering at $B=0, \beta(g)$ is approximated by $\beta_{o}$ for $L \lesssim \ell_{\text {so }}$, and by $\beta_{s}$ for $L \gtrsim \ell_{\text {so }}\left(\ell_{\text {so }}\right.$ is the length of spin-orbit scattering).

The above assumption can be easily used in the RG procedure to estimate the change of $\xi$ as a function of $B$. Let us start at $d \leq 2$ for weak disorder, $k_{F} \ell \gg 1$, where localization still exists for large $L$. We start at a length scale $L_{0}$, satisfying $\ell \lesssim L_{0} \ll \xi$, where $g \gg 1$ and integrate the RG equation until getting to the regime where $g \sim 1$. This regime corresponds to the scale $L \sim \xi$, and this is how $\xi$ is determined.

We approximate $\beta(g)$ by the weak-localization expressions [12,13]:

$$
\begin{aligned}
& \beta_{o}(g)=d-2-1 / g, \\
& \beta_{u}(g)=d-2-1 / 2 g^{2}, \\
& \beta_{s}(g)=d-2+1 / 2 g .
\end{aligned}
$$

The exact matching of $\beta(g)$ at $L \sim \ell_{H}$ or $L \sim \ell_{\text {so }}$ yields only unimportant corrections. We do not consider the case where both time-invariance and spin-rotations symmetries are broken.

We begin with a quasi $1 D$ wire, having $N_{\perp}$ conduction channels (i.e. $N_{\perp}$ transfer states below $E_{F}$ ). For the wire of a $2 D$ cross section the classical Drude conductance is given by

$$
g_{c \ell}(L)=\frac{\pi}{3} N_{\perp} \frac{\ell}{L} .
$$

We start integration of Eq. (11) from a small $L_{0} \ll \xi$ (but $L_{0} \gg \ell$ ). For $B$ large enough, $\ell_{H} \lesssim \xi_{o}$ ( $\xi_{o}$ is the $B=0$ localization length), we first integrate Eq. (1) in the orthogonal symmetry range, from $L_{0}$ to $\ell_{H}$, with the $\beta$-function $\beta_{o}$, Eq. (2a), to obtain (using $g_{c \ell}\left(L_{0} \gg 1\right.$ )

$$
g(L)+1=g_{c \ell}(L), \quad L \lesssim \ell_{H} .
$$

Note that $L_{0}$ has cancelled out. For $L$ 's above $\ell_{H}$, starting with $L=\ell_{H}$ we integrate Eq. (11) with the $\beta$-function $\beta_{u}$, Eq. (2b), to find (using $g\left(\ell_{H}\right) \gg 1$ )

$$
g^{2}(L)+1 / 2=g_{c \ell}^{2}(L), \quad L \gtrsim \ell_{H} .
$$

Note that $\ell_{H}$ has cancelled out as well, this will be seen to be the source of the universal increase of $\xi$ with B in the whole regime considered in the quasi $1 D$ case.

For a very weak magnetic field, $\ell_{H} \gg \xi_{o}$, Eq. (4) is valid in the whole range of $L$, up to $\xi_{o}$, and the behavior is "orthogonal" throughout, still allowing small $B$-dependent

of account interesting effects that may occur in the range of even higher fields, $\ell_{H}{ }^{2} \lesssim \ell / k_{F}$, where one has to scale $g_{x x}$ and $g_{x y}$ together 11. corrections to $\xi$. At $L=\xi_{o}, g\left(\xi_{o}\right)=A, A$ being a constant of order unity. Thus

$$
\xi_{o}=\frac{\pi}{3} \frac{N_{\perp} \ell}{A+1},
$$

in accordance with the well-known Thouless result [14]. In the purely unitary case, i.e. for a "strong" magnetic fields $\ell_{H} \sim L_{0}$, one has an increase of $\xi$ :

$$
\xi_{u}=\frac{\pi}{3} \frac{N_{\perp} \ell}{\sqrt{A^{2}+1 / 2}} .
$$

A similar increase takes place in the presence of spin-orbit scattering. Repeating the same procedure as above, one finds that for the purely symplectic case

$$
\xi_{s}=\frac{\pi}{3} \frac{N_{\perp} \ell}{A-1 / 2}
$$

While the precise value of $\xi_{u} / \xi_{o}$ and $\xi_{s} / \xi_{o}$ cannot be determined by this simple approach, this increase is qualitatively due to $\beta(g)$ being larger in the unitary case, and even more so in the symplectic case so that one has to go to larger scales to get $g \sim 1$. Since $\ell_{H}$ dropped from Eq. (5), the increase from $\xi_{0}$ to $\xi_{u}$ is universal (i.e. it does not depend on $B$ ) in the whole range $\ell \lesssim \ell_{H} \lesssim \xi$. Similarly, the increase of $\xi$ in the symplectic case does not depend on the spin-orbit scattering strength for $\ell \lesssim \ell_{\text {so }} \lesssim \xi$. Thus our scaling consideration, while not able to yield the constants, is consistent with the exact result [5] that in a quasi $1 D$ metallic wire there is an increase of $\xi$ in the appropriate, rather wide, range of $B$.

The physical reason for the increase of $\xi$ with $B$ is now very clear. It is due to the well-known delocalization for the weakly localized regime $L \ll \xi_{o}$, caused by the elimination of coherent time-reversed paths by the magnetic field $B$. The scaling procedure enables us to approximately carry this effect all the way up to $L \sim \xi$ and see by how much $\xi$ is changing.

The same procedure which leads to semi-quantitatively correct results in quasi $1 \mathrm{D}$ and illuminates the physics of delocalization by the field, can be repeated for a $2 D$ film. We start here at $L_{0} \sim \ell$ with a conductance $g_{0}$ and obtain for $\xi_{o} \gtrsim \ell_{H} \gtrsim \ell$

$$
\xi=\ell_{H} \exp \left(g_{H}^{2}\right)
$$

where $g_{H} \equiv g\left(\ell_{H}\right)=g_{0}-\ln \left(\ell_{H} / \ell\right)$. This ranges from the orthogonal value given by $g_{0} \sim \ln \left(\xi_{o} / \ell\right)$, i.e. $\xi_{o} \cong \ell e^{g_{0}}$ for $\ell_{H} \gtrsim \xi_{o}$, to the unitary one, $\xi_{u} \cong \ell e^{g_{0}^{2}}$ for $\ell_{H} \sim \ell$. Since $g_{0} \gg 1, \xi_{u}$ is overwhelmingly larger than $\xi_{o}$. The ratio $\xi_{u} / \xi_{o}$ is obviously not universal - it changes by many orders of magnitude in the range $\ell \lesssim \ell_{H} \lesssim \xi_{o}$.

In the symplectic case, the $\beta_{s}$ function, Eq. (2d), is always positive in the weak localization regime. So for $\xi_{o}<\ell_{\mathrm{so}}$, there is an "antilocalization" effect: the conductance is decreasing with increasing the scale up to $L \sim \ell_{\text {so }}$. Then the $\beta$ function in Eq. (11) changes sign 
from negative, Eq. 2a), to positive, Eq. (2c), and $g$ begins to increase. The antilocalization character of the $\beta$ function might change at $g \sim 1$ due to further terms of the $1 / g$ expansion 15]. Such a change, if any, is definitely beyond the scope of the present treatment.

We now consider the situation for $d>2$, where the Anderson transition exists at a certain $g=g_{c}$. Here we use the RG equation (11) linearized near $g_{c}\left(\right.$ for $g-g_{c} \ll 1$ ) with

$$
\beta(g)=\frac{1}{\nu} \frac{g-g_{c}}{g_{c}},
$$

where $\nu$ is the exponent of the diverging $\xi$ near the transition. Indeed, the solution to Eq. (11) with the linearized $\beta$-function (10) for $g(=g(L))$ at a general scale $L$ is written as:

$$
g_{c}-g=\left(g_{c}-g_{0}\right)\left(L / L_{0}\right)^{1 / \nu},
$$

where $g_{0}=g\left(L_{0}\right)$. We rewrite this in the localized phase $\left(g_{0}<g_{c}\right)$ as

$$
g=g_{c}\left[1-(L / \xi)^{1 / \nu}\right], \quad \xi \equiv L_{0}\left(1-g_{o} / g_{c}\right)^{-\nu} .
$$

From now on we take the above notation, with the constants $g_{c}$ and $\nu$, to describe the orthogonal case $(B=0)$ with $\xi=\xi_{o}$. The unitary ("large $B$ ") case satisfies the same scaling theory but with the constants $g_{c}$ and $\nu$ replaced by $g_{c H}$ and $\nu_{H}$. In the $\epsilon$ expansion for $d=2+\epsilon$, the transition at small $\epsilon$ occurs in a range describable by perturbation theory, and we can use the weak-localization expressions (2) to obtain the following constants

$g_{c}=1 / \epsilon, \nu=1 / \epsilon ; g_{c H}=(1 / 2 \epsilon)^{1 / 2}, \quad \nu_{H}=1 / 2 \epsilon$.

We shall assume that $g_{c H}<g_{c}$ also at $\epsilon=1$ and use our ansatz that the system behaves according to orthogonal or unitary symmetry for $L<\ell_{H}$ or $L>\ell_{H}$ respectively. We shall also assume that the crossover is describable by the linearized $\beta(g)$. The latter assumption, too, is justified in the $\epsilon$-expansion. We do the considerations below for a general $d>2$. They are certainly valid for $\epsilon \ll 1$ and should be qualitatively correct at $d=3$. (Unfortunately, such an approach is not feasible for the spin-orbit case where $g_{c}$ is not large even for small $\epsilon$, and the $\epsilon$ expansion could hardly provide with useful insight into the $3 D$ case, besides the fact, though, that the spin-orbit interaction still does cause delocalization for $\ell \ll \ell_{\text {so }} \ll \xi$ in the weakly localized phase near the transition.)

Again, for $\ell \lesssim \ell_{H} \lesssim \xi$, we use the orthogonal results, Eqs. (11), (12), from $L_{0}=\ell$ to $\ell_{H}$ :

$g\left(\ell_{H}\right)=g_{c}-\left(g_{c}-g_{0}\right)\left(\ell_{H} / \ell\right)^{\frac{1}{\nu}}=g_{c}\left[1-\left(\ell_{H} / \xi_{o}\right)^{\frac{1}{\nu}}\right]$

At the scale $L \gtrsim \ell_{H}$, we use the unitary results. When $g\left(\ell_{H}\right)>g_{c H}$, the decrease in $g$, Eq. (14), is changed by an increase as the unitary $\beta$-function (10) is positive at this scale. Then, the critical conductance as function of $B$,
$g_{c}(B)$, is given by $g_{0}$ for which $g\left(\ell_{H}\right)=g_{C H}$. Substituting into this equation $g\left(\ell_{H}\right)$, Eq. (14), and solving for $g_{0}=$ $g_{c}(B)$ we find:

$$
g_{c}(B)=g_{c}-\left(g_{c}-g_{c H}\right)\left(\ell / \ell_{H}\right)^{\frac{1}{\nu}}
$$

To check this, we see that $g_{c}(B)=g_{c H}$ for $\ell_{H}=\ell$, and $g_{c}(B) \rightarrow g_{c}$ for $\ell_{H} \rightarrow \infty(B \rightarrow 0)$. The real divergence of $\xi$ near the transition occurs for a given $B$ when $g_{c}(B) \rightarrow$ $g_{0}$ :

$$
\begin{aligned}
\xi_{H} & =\ell_{H}\left(1-\frac{g\left(\ell_{H}\right)}{g_{c H}}\right)^{-\nu_{H}} \\
& =\ell_{H}\left(\frac{\ell_{H}}{\ell}\right)^{-\nu_{H} / \nu}\left(\frac{g_{c}(B)-g_{0}}{g_{c H}}\right)^{-\nu_{H}}
\end{aligned}
$$

At this scale, $g$ is given by Eq. (12) with substituting $g_{c H}, \xi_{H}$, and $\nu_{H}$ for respectively $g_{c}, \xi_{o}$, and $\nu$. Clearly, there is nothing universal in the ratio of $\xi_{H} / \xi$. A similar conclusion for $d=2,3$, both for the magnetic and spin-orbit case, has been made in Ref. 16] based on the heuristic picture of localization of Ref. [17.

The most important aspect of delocalization by a magnetic field in the range considered $\left(\ell \lesssim \ell_{H} \lesssim \xi_{o}\right)$ at $d>2$ is the shift of the transition towards delocalization. This is seen from the fact that for small $\epsilon, g_{C H} \ll g_{c}$ and it is expected that $g_{c H}<g_{c}$ also at $d=3$. For a given field B there are three regimes: for $g \leq g_{c}(B)$, there is a localization also with the magnetic field but $\xi$ is larger (arbitrarily larger near $g_{c}(B)$ ); for $g_{c}(B) \leq g \leq g_{c}$ the magnetic field destroys localization completely; for $g>g_{c}$, there is no localization but the correlation length $\xi$ appears to be larger at $B=0$ (especially just above $g_{c}$ ). The shift of the transition with field has been discussed in Refs. [18,19]. Clearly, the effect of $B$ for $d \geq 2$ is not universal except, of course, that the asymptotic critical behavior is governed by the unitary universality class for any $B)$. It is only for quasi 1D systems that $\xi$ increases by a universal factor in the whole range $\ell \lesssim \ell_{H} \lesssim \xi_{o}$. It is interesting that the considerations based just on the symmetry breaking within the transfer matrix approach [7] seem to be valid only for quasi 1D systems. They had, however, highlighted the very interesting and at the time almost unexpected possibility of substantial delocalization by the magnetic field.

The scaling cross-over considerations exemplified here can be applied to various physical situations involving the phase-breaking length $\ell_{\phi}$, alongside with $\ell_{H}$ and $\ell_{\mathrm{so}}$ in appropriate ranges of the localized phase. This produces a variety of interesting magnetoconductance phenomena in the localized phase.

\section{ACKNOWLEDGMENTS}

The authors thank Y. Gefen, O. Entin-Wohlman, D.E. Khmelnitskii, Z. Ovadyahu, U. Sivan and U. Smilansky 
for discussions on this and related topics. We are grateful to the Wissenschaftskolleg in Berlin for kind hospitality extended to us at the initial stage of this work. This work was supported by the Minerva Foundation, by the German-Israeli Foundation (GIF), and by the EEC grant No. SSC-CT90-0020.

[1] F. J. Dyson, J. Math. Phys. 3, 140 (1962).

[2] E. Abrahams, P. W. Anderson, D. C. Licciardello, and T. V. Ramakrishnan, Phys. Rev. Lett. 42, 673 (1979).

[3] F. Wegner, Z. Phys. B 35, 207 (1979).

[4] See for reviews: P. A. Lee and T. V. Ramakrishnan, Rev. Mod. Phys. 57, 287 (1985); B. L. Altshuler and A. G. Aronov, in Electron-Electron Interactions in Disordered Systems, p. 1, edited by A. L. Efros and M. Pollak, NorthHolland, Amsterdam (1985).

[5] K. B. Efetov and A. I. Larkin, Zh. Eksp. Teor. Fiz. 85, 764 (1983) [Sov. Phys. JETP 58, 444 (1983)]; K. B. Efetov, Adv.Phys. 32, 53 (1983).

[6] O. N. Dorokhov, Zh. Eksp. Teor. Fiz. 85, 1040 (1983) [Sov. Phys. JETP 58, 606 (1983)].

[7] A. D. Stone, P. A. Mello, K. A. Muttalib, and J.-L. Pichard, in Mesoscopic Phenomena in Solids, p. 369, edited by B. L. Altshuler, P. A. Lee, and R. A. Webb, North-Holland, Amsterdam, (1991).

[8] J.-L. Pichard, M. Sanquer, K. Slevin, and P. Debray, Phys. Rev. Lett. 65, 1812 (1990); K. Slevin, J.-L. Pichard, and K. A. Muttalib, Journal de Physique 3, 1387 (1993).

[9] M. L. Mehta, Random matrices (Academic Press, Boston, 1991).

[10] M. Thaha, R. Blümel, and U. Smilansky, Phys. Rev. E 48, 1764 (1993).

[11] D. E. Khmelnitskii, Pis'ma v ZhETF 38, 454 (1983) [JETP Letters 38, 552 (1983)]; Phys. Lett. 106A, 1097 (1984).

[12] E. Brézin, S. Hikami, and J. Zinn-Justin, Nucl. Phys. B 165, 528 (1980).

[13] F. Wegner, Phys. Repts. 67, 15 (1980).

[14] D. C. Thouless, Phys. Rev. Lett. 39, 1167 (1977).

[15] F. Wegner, Nucl. Phys. B 316, 663 (1989).

[16] J. P. Bouchaud, J. Phys. 1 (France) 1, 985 (1991); J. P. Bouchaud and D. Sornette, Europhys. Lett. 17, 721 (1991).

[17] D. Sornette, and B. Souillard Europhys. Lett. 13, 7 (1990).

[18] A. I. Larkin and D. E. Khmelnitskii, Sol. State Commun. 39, 1069 (1981).

[19] B. Shapiro, Phil. Mag. B50, 241 (1984). 